\title{
Lossy Image Compression Using Discrete Wavelet Transform and Thresholding Techniques
}

\author{
V. Alarcon-Aquino ${ }^{*}, 1$, J. M. Ramirez-Cortes ${ }^{2}$, P. Gomez-Gil ${ }^{2}$, O. Starostenko ${ }^{1}$ and \\ H. Lobato-Morales ${ }^{2}$
}

${ }^{1}$ Universidad de las Americas Puebla, Department of Computing, Electronics and Mechatronics, Sta. Catarina Martir, Cholula, Puebla, 72810, Mexico

${ }^{2}$ National Institute of Astrophysics, Optics and Electronics, Department of Electronics and Computer Science, Tonantzintla, Puebla, Mexico

\begin{abstract}
Results of lossy image compression using wavelet transforms and several thresholding techniques are presented here. The analyzed image was divided into little sub-images and each one was decomposed in a vector following a Hilbert fractal curve. The wavelet transform was applied to each vector and some of the high frequency components were suppressed based on some threshold criteria. Different levels of wavelet decomposition and wavelet mother functions were assessed. The Huffman coding algorithm was then applied in order to reduce image weight. Simulation results have revealed that high compression ratios were obtained with the mean and the standard deviation thresholding algorithms at different levels of wavelet decomposition.
\end{abstract}

Keywords: Discrete wavelet transform, image compression, Hilbert curve, thresholding techniques, Huffman coding.

\section{INTRODUCTION}

The growth of multimedia applications has led to the development of new techniques for image compression and image de-noising. Standardized image compression formats as JPEG use sub-images decomposition, bi-dimensional discrete cosine transform (DCT) of each one, and the Huffman coding algorithm [1, 2]. Some of the high frequency components are eliminated at different ratios keeping only the greater energy components, and then a codification method based on redundancy is applied. This coefficient suppression generates a lossy image that is several times undetectable by the human visual system (HVS). Several spectral transforms have also been reported, including Fourier, Walsh, Hadamard, and Hartley transforms [2], and have shown that better results are obtained with wavelet transforms [2-4]. An image compression-based application using wavelet transforms has been reported where the authors have shown the performance of their approach in multimedia data exchange for mobile devices using Japanese kanji or Mayan glyphs images [4]. The last JPEG version, JPEG 2000, uses the discrete wavelet transform (DWT) instead of DCT, allowing higher peak signal-to-noise ratio (PSNR) and compression ratios $\left(\mathrm{R}_{\mathrm{c}}\right)$. Recently, several approaches have been proposed for data compression based on wavelet decomposition [2-7].

*Address correspondence to this author at the Department of Computing, Electronics and Mechatronics, Sta, Catarina Martir, Cholula, Puebla, 72810, Mexico; Tel: +52 (222) 2292042; Fax: +52 (222) 2292138;

E-mail: vicente.alarcon@udlap.mx
In this paper, we propose an approach for image compression based on the DWT using different types of wavelets and thresholding techniques. The first step of the proposed compression algorithm is image decomposition in $8 \times 8$ sub-images. Each one is then converted to a 64-point vector following a scan with a Hilbert Fractal curve. The wavelet transform is thus applied to each vector and some of the high frequency components are suppressed based on some threshold criteria.

\section{WAVELET TRANSFORMS AND THRESHOLDING TECHNIQUES}

Wavelet transforms involve representing a general function in terms of simple, fixed building blocks at different scales and positions. These building blocks are generated from a single fixed function called mother wavelet by translation and dilation operations [8]. The mother wavelet function $\psi(t)$, scaling $\mathrm{a}_{0}$ and translation $\mathrm{b}_{0}$ parameters are specifically chosen such that $\psi(t)$ constitutes orthonormal bases for $L^{2}(\Re)$, where $\Re$ denotes real numbers $[9,10]$. To form orthonormal bases with good time-frequency localization properties, the time-scale parameters $(b ; a)$ are sampled on a so-called dyadic grid in the time-scale plane, namely, $a_{0}=2$ and $b_{0}=1$ [9]. A formal approach to constructing orthonormal bases is provided by multiresolution analysis (MRA). The idea of MRA is to write a function $x(t)$ as a limit of successive approximations, each of which is a smoother version of $x(t)$. The successive approximations thus correspond to different resolutions. This process is also known as sub-band coding [2]. As stated 
previously, the wavelet transform is applied to a 64-point vector of each sub-image that follows a Hilbert fractal curve and some of the high frequency components are suppressed based on some threshold criteria. Several thresholding techniques have been reported [11]. For high frequency coefficients suppression, a threshold level must be obtained in order to eliminate only the lower energy contents of the data details and consequently achieving low image distortion. Thresholding techniques can be classified into hard and soft threshold [12]. The former eliminates the coefficients under a given threshold and maintains the other at the original values, while the latter is an extension of the former, but has one difference, the coefficients below the threshold are eliminated and the other are setting to a new value [12]. In the work reported here, four algorithms are used to obtain the threshold value, namely, minimax, fixed form, rigorous sure, and heuristic sure [11]. All of these based mainly on the number of samples of the transformed signal. These thresholding algorithms are developed for image de-noising and are applied here for image compression. Note that for comparison purposes, two more thresholding algorithms are assessed, namely, the mean and the standard deviation leading the threshold as the mean of the absolute value of the wavelet coefficients in the former and the standard deviation of wavelet coefficients in the latter.

\section{PROPOSED APPROACH}

In what follows, the approach to image compression based on the discrete wavelet transform is proposed. Due to special characteristics on the human visual system, it is possible to distort the image without significant degradation [13]. Accordingly, some of the high frequency coefficients can be suppressed thereby achieving good compression performances. The first step of the proposed compression algorithm is image decomposition in $8 \times 8$ sub-images. Each one is then converted to a 64-point vector following a scan with a Hilbert fractal curve as shown in Fig. (1) [2]. The main impetus in using this fractal curve is to follow a more distributed pattern. The resultant vectors are located in a new matrix called TE with size $p \times q$ where

$$
p=m \times n / 64
$$

and $q=64$. The values $m$ and $n$ in (1) compose the original image dimensions.

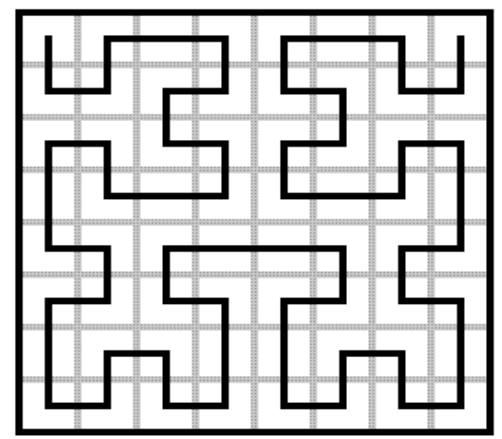

Fig. (1). Standard scan sub-image.
A 1-D Discrete Wavelet Transform is realized to each row of the TE matrix $\left(\mathbf{T E}_{i}=c_{j-1, m}^{i}\right)$, and the resulting scaling and wavelet coefficients ( $c_{j, n}^{i}$ and $d_{j, n}^{i}$, respectively) form the correspondent of the $i$-th row-vector $\mathbf{T F}_{i}$ of the new transformed matrix $\mathbf{T F}$ :

$$
\begin{aligned}
c_{j, n}^{i} & =\sum_{l \in Z} g_{l} c_{j-1,2 n-l}^{i} \\
d_{j, n}^{i} & =\sum_{l \in Z} h_{l} c_{j-1,2 n-l}^{i}
\end{aligned}
$$

$i=1,2, \ldots, m \times n / 64 ; j=1,2, \ldots, J$

where $J$ corresponds to the sub-band coding level of the DWT, $c_{j-1,2 n-l}^{i}$ is the $i$-th row of the TE matrix, and $g_{l}$ and $h_{l}$ are the low-pass and high-pass filters, respectively used for the DWT. High frequency coefficients are suppressed based on each thresholding criteria mentioned previously following the hard-threshold or soft-threshold scheme. An example of a vector thresholding is shown in Fig. (2). It is proposed here that at this step, the Huffman coding algorithm is implemented. Huffman coding is an entropy coding algorithm used for lossless data compression [1]. This symbol probability-based method forms new code-words in order to remove data redundancy whereby the zero columns of the TF matrix allows greater compressed data due to the 0 symbol repetition.

When encoding the symbols, Huffman coding yields the smallest possible number of code symbols per source symbol. The first step in Huffman coding is to create a series of source reduction by ordering the probabilities of the symbols under consideration [1]. The greatest probability symbol is replaced by a single symbol code. The largest coding symbol corresponds to the lowest probability source symbol. Then a "dictionary" is created from the source data. Each generated code must start with " 1 " and ends with " 0 ", except for the greatest probability symbol, which is only represented by a " 0 ". Using this dictionary, a coding vector is created from the original data. An example of Huffman coding dictionary is shown in Table 1. If it was assumed a data vector $\mathbf{A}=\{000,000,001,101\}=\left\{A_{2} A_{1} A_{2} A_{6}\right\}$, then the corresponding encoding vector is $\mathbf{H}=[0110010]$. In this case, $A_{n}$ is composed by three bits, which clearly shows that the new vector is lower than the source vector. Using high dimension data this encoding algorithm yields great compression values. The decoding process is the inverse codification and is obtained directly, based on the dictionary codes.

To reconstruct the image, the inverse process of decoding data from the Huffman algorithm forms again the TF matrix. The Inverse Discrete Wavelet Transform (IDWT) is applied to each row $\mathbf{T F}_{i}$ of the transformed matrix. The IDWT process is then obtained in the same way as the DWT using the scaling and wavelet coefficients, the same filters, and the same mother wavelet function utilized for the direct transformation:

$$
\begin{aligned}
& \mathbf{T E}_{i}^{s}=c_{j-1, n}^{i}=\sum_{l \in Z} g_{l} c_{j, 2 n-l}^{i}+\sum_{l \in Z} h_{l} d_{j, 2 n-l}^{i} \\
& i=1,2, \ldots, m \times n / 64, j=1,2, \ldots, J
\end{aligned}
$$


a) Transformed vector.

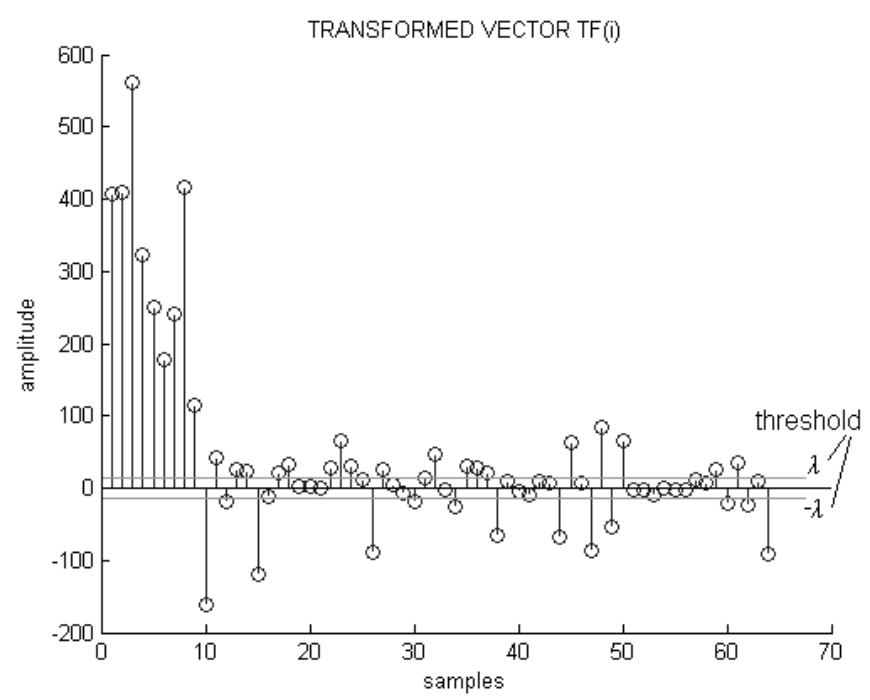

b) Vector with suppressed coefficients.

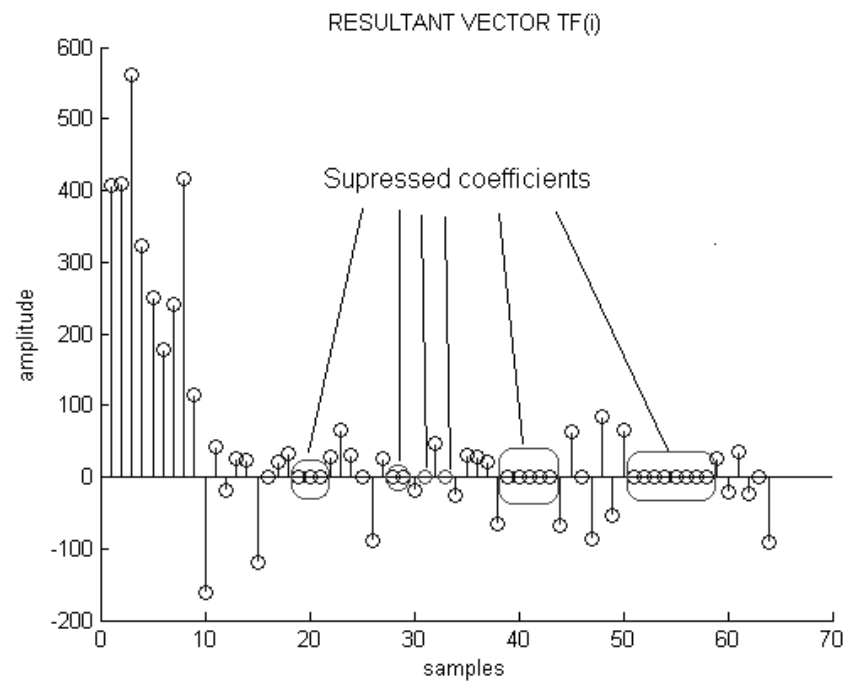

Fig. (2). An Example of thresholding.

Table 1. Huffman Coding Dictionary

\begin{tabular}{|c|c|c|}
\hline Symbol & Prob & Code \\
\hline \hline$A_{2}$ & 0.4 & 0 \\
\hline$A_{6}$ & 0.3 & 10 \\
\hline$A_{1}$ & 0.1 & 110 \\
\hline$A_{4}$ & 0.06 & 1110 \\
\hline$A_{3}$ & 0.05 & 11110 \\
\hline$A_{5}$ & 0.05 & 111110 \\
\hline$A_{8}$ & 0.02 & 1111110 \\
\hline$A_{7}$ & 0.02 & 1111111 \\
\hline
\end{tabular}

The matrix $\mathbf{T E}^{s}$ is reconstructed from the IDWT of each of the row-vectors of $\mathbf{T F}$. Then the 64-point rows of $\mathbf{T E}_{i}^{s}$ reconstruct the correspondent sub-images following the same Hilbert fractal curve and thus generating the new image.

\section{SIMULATION RESULTS}

Here, we report simulation results of the proposed approach using several wavelets and different thresholding techniques. A $256 \times 256$ pixels image of Lena is decomposed into $8 \times 8$ sub-images and each one is translated to a $\mathbf{T E}_{i}$ vector following the mentioned Hilbert fractal curve. In this case, $i=1,2, \ldots, 1024$ and therefore a $1024 \times 64$ TE matrix is then generated. A 5-level DWT is applied to each of the TE rows, forming the TF matrix, which for some cases (depends on the wavelet mother function used) is the same size as the TE matrix. High frequency coefficients are suppressed based on one of the thresholding criteria: high frequency mean, high frequency standard deviation, minimax, fixed form, rigorous sure, and heuristic sure and results are shown for comparison. A noise standard deviation of 1 is assumed. The Huffman coding algorithm is then applied to the TE matrix in order to achieve data compression. The peak signal-tonoise ratio (PSNR) is used to assess the performance of the proposed approach. The PSNR is calculated according to

$$
\operatorname{PSNR}(d B)=10 \log _{10}\left(\frac{255^{2}}{M S E}\right)
$$

where $M S E$ is the mean square error defined by

$$
M S E=\frac{\sum_{x=0}^{m-1} \sum_{y=0}^{n-1}[\hat{f}(x, y)-f(x, y)]^{2}}{m \cdot n}
$$

where $f(x, y)$ and $\hat{f}(x, y)$ correspond to the original image and resulting image values respectively, $x$ and $y$ are the spatial coordinates, and $m$ and $n$ are the total image dimensions. The compression ratio $R_{C}$ is simply obtained by

$$
R_{C}=\frac{m \cdot n \cdot 8}{\text { coded bits }} .
$$

Fig. (3) shows the PSNR (dB) for each of the six thresholding algorithms. A Daubechies1 (Haar) mother wavelet (db1), a 5-level of decomposition, and a $256 \times 256$ size image of Lena are used. The figure also compares the hard and soft threshold schemes. Fig. (4) plots the PSNR versus its corresponding $R_{C}$ values for both the hard and soft thresholding schemes.

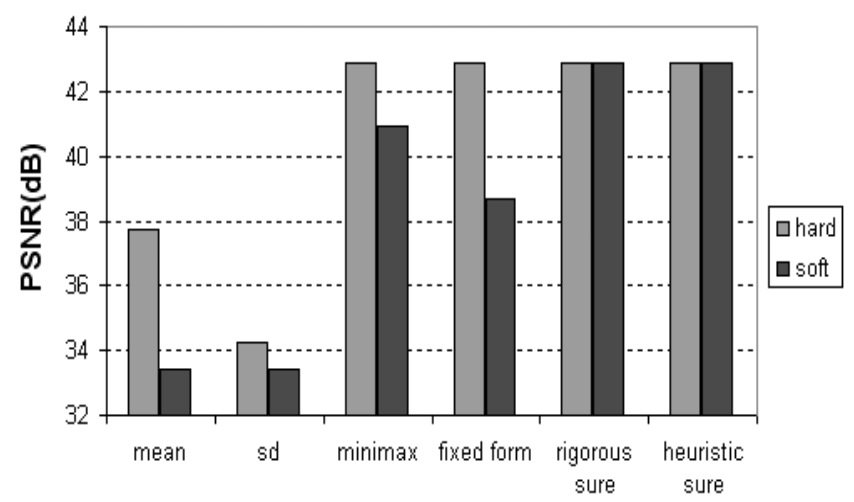

Fig. (3). The values of PSNR (dB) for hard and soft threshold.

The soft threshold scheme generates slightly higher $R_{C}$ compared to that of hard threshold, but the main 
disadvantage is that the PSNR decreases, as shown in Figure 4. Numerical results for the hard threshold scheme are shown in Table 2. It can be seen that good compression values are achieved when the mean and the standard deviation thresholding algorithms are used; nonetheless, they generate higher image distortion. These results are comparable with [13] where the authors reported PSNR $=32.66 \mathrm{~dB}$ and $\mathrm{Rc}=4.25$ using a modified version of the Lempel-Ziv algorithm. Note that this algorithm allows additional compression ratio but it generates greater image distortion [13]. Fig. (5) shows $R_{C}$ and PSNR (dB) using 2, 3, 4 and 5 levels of wavelet decomposition. It is observed that the higher number of sub-bands used, the higher compression ratio is obtained without modifying image distortion significantly. A comparison using other wavelets like Daubechies 2, Symmlet 3, and Coiflet 1, is presented in Fig. (6), and compared with the initial one (Daubechies1 or Haar). Five levels of wavelet decomposition and a hard threshold schemes were used. Note that the variations of $R_{C}$ and PSNR due to the different type of wavelets are mainly because the mother wavelet functions generating different transformed vector lengths. It is important to note here that when using these thresholding algorithms, the simpler wavelet is used, better performance was obtained.

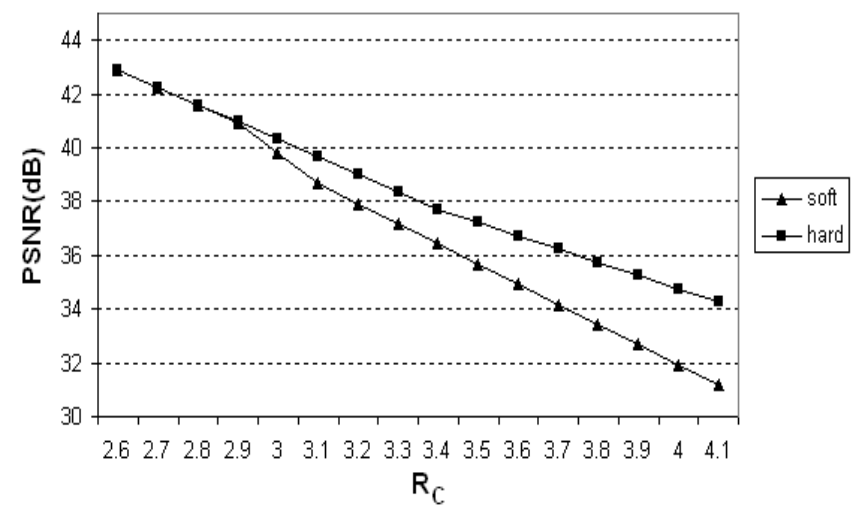

Fig. (4). The values of PSNR (dB) vs $\mathbf{R}_{\mathrm{C}}$.

Table 2. Compression Results

\begin{tabular}{|c|c|c|c|}
\hline & PSNR(dB) & MSE & $\mathbf{R}_{\mathbf{C}}$ \\
\hline \hline Mean & 37.73 & 10.97 & 3.37 \\
\hline Std dev & 34.25 & 24.43 & 4.09 \\
\hline Minimax & 42.9 & 3.33 & 2.57 \\
\hline Fixed form & 42.86 & 3.37 & 2.61 \\
\hline Rigorous sure & 42.9 & 3.33 & 2.57 \\
\hline Heuristic sure & 42.9 & 3.33 & 2.57 \\
\hline
\end{tabular}

Note that the selection of a wavelet must be related to the common features of the events present in real signals. That is, a wavelet should be well adapted to the events to be analyzed [8] and the design of a wavelet must be optimized to produce a maximum number of wavelet coefficient that are close to zero. This particular feature is not only important for data compression but also for noise removal and fast calculations [10]. Different wavelet families have a trade-off between the degree of symmetry (i.e., linear phase characteristics of wavelets) and the degree to which ideal high-pass filters are approximated $[9,10]$. The degree of symmetry in a wavelet is important in reducing the phase shift of features during the wavelet decomposition. If the phase shift is large, it can lead to distortions in the location of features in the transform coefficients. The degree to which ideal high-pass filters are approximated is also important since, ideally, the wavelet filter should resemble a high-pass $(j=1)$ or band-pass filter $(j>1)$, while the scaling filter should resemble a low-pass filter. For example, the Haar wavelet has compact support; however, it has just one vanishing moment and is piece-wise constant. Furthermore, the resulting wavelet basis functions have the characteristic of being discontinuous, which renders them unsuitable as basis functions for classes of smoother functions.

a) $R_{C}$

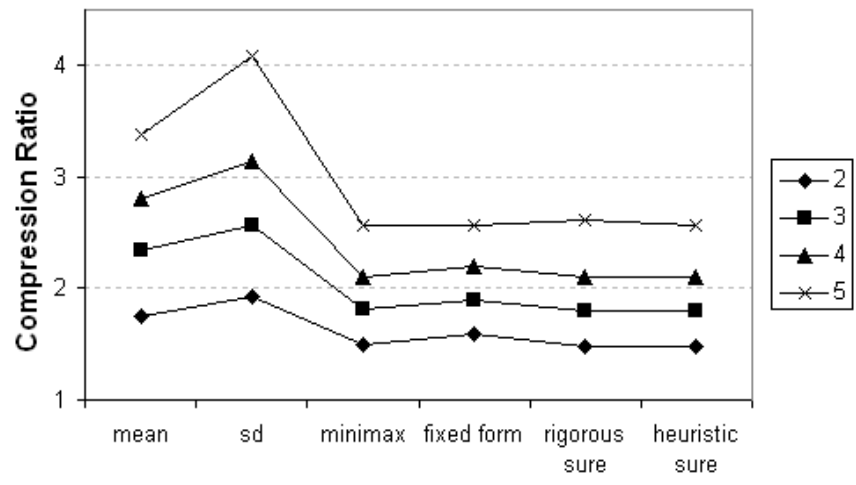

b) PSNR (dB)

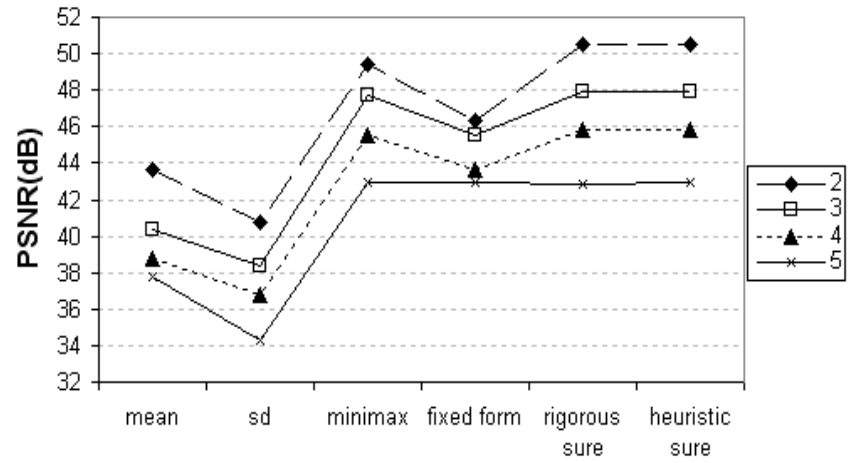

Fig. (5). The values of a) $R_{C}$, and b) PSNR (dB) for 2, 3, 4 and 5 levels of decomposition.

To overcome the disadvantage of the Haar wavelet, Daubechies has developed a theory for obtaining higher order mother wavelet filters with compact support [9]. These filters have even lengths $L$ (between 2 and 20); however, they are identified not by the length $\mathrm{L}$ but by the number of vanishing moments, $v=L / 2$. As the number of vanishing moments increases, the wavelet filter becomes longer and its approximation to an ideal high-pass filter improves. That is, a lesser amount of leakage is obtained, which is caused by the non-ideal filter shape [8]. Daubechies proved that the Haar filter is the only real compactly supported conjugate mirror filter that has a linear phase. The Symmlet filters of 
Daubechies are optimized to obtain an almost linear phase and they are more symmetric. On the other hand, Coiflet filters have $v$ vanishing moments and a minimum size support [10]. The order of the filter is equal to the number of vanishing moments and, for the case, of Daubechies filters, it is half the length of the filter. Note that for $v=1$, the Daubechies wavelet filter is equivalent to the Haar wavelet filter.

Figs. (8, 9) show the resulting images using standard deviation and rigorous sure thresholding algorithms, respectively. Both of them based on hard threshold scheme, 5-level decomposition and using wavelet $d b 1$. For comparison purposes, the original image is shown in Fig. (7) as well. The mean and standard deviation thresholding algorithms generate similar reconstructed images and PSNR in which the differences between them are slightly noticeable by HVS. Hence, only the resulting image from the standard deviation algorithm is shown here. Reconstructed images using minimax, fixed form, rigorous sure and heuristic sure algorithms have lower distortion compared to that of mean and standard deviation algorithms, and consequently not noticeable by the HVS when compared to the original image. In addition, their PSNR values are very similar, and therefore only the image generated by using rigorous sure algorithm is shown here.

a) $R_{C}$

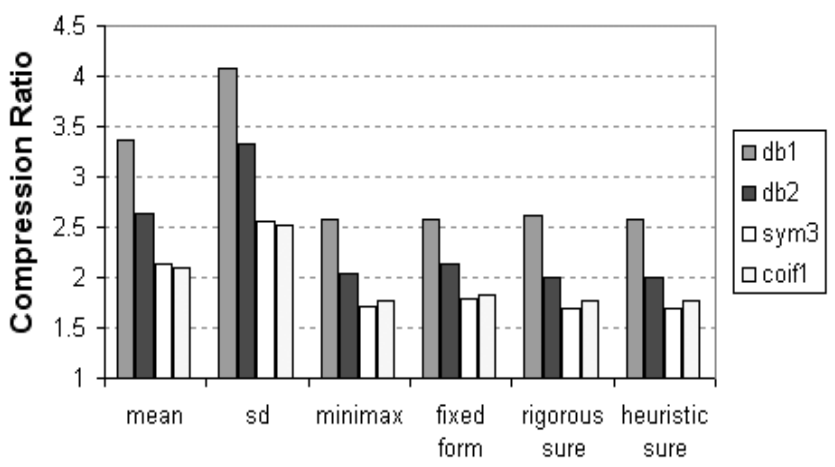

b) $\operatorname{PSNR}(\mathrm{dB})$

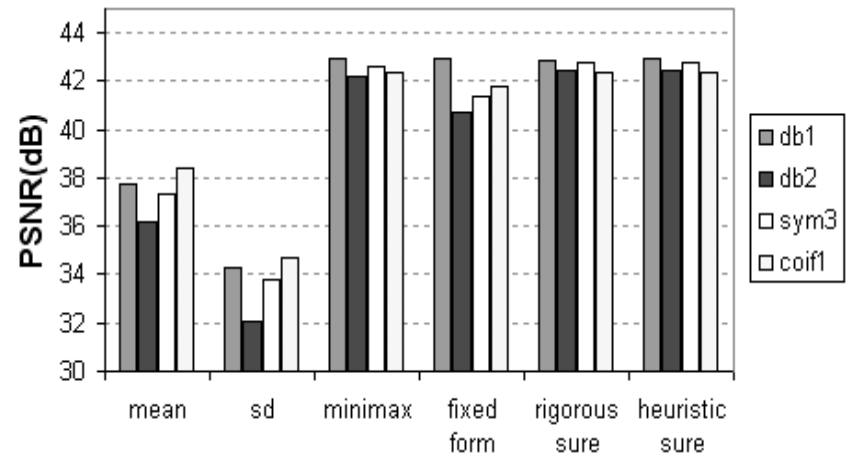

Fig. (6). The values of a) $R_{C}$, and b) PSNR (dB) for different types of wavelets.

Finally, the error images generated by standard deviation and rigorous sure thresholding algorithms are shown in Figs. $(\mathbf{1 0}, \mathbf{1 1})$, respectively. It is clearly observed from Fig. (10) that standard deviation thresholding algorithm introduces a high level of distortion due to the elimination of many high frequency coefficients. By using this thresholding technique, only the lowest details are suppressed, and consequently a sense of border detection can be appreciated in its respective error image. When minimax, fixed form, rigorous sure and heuristic sure algorithms are applied to image compression, a better de-noised image is observed. In fact, its corresponding error images are mainly noise.

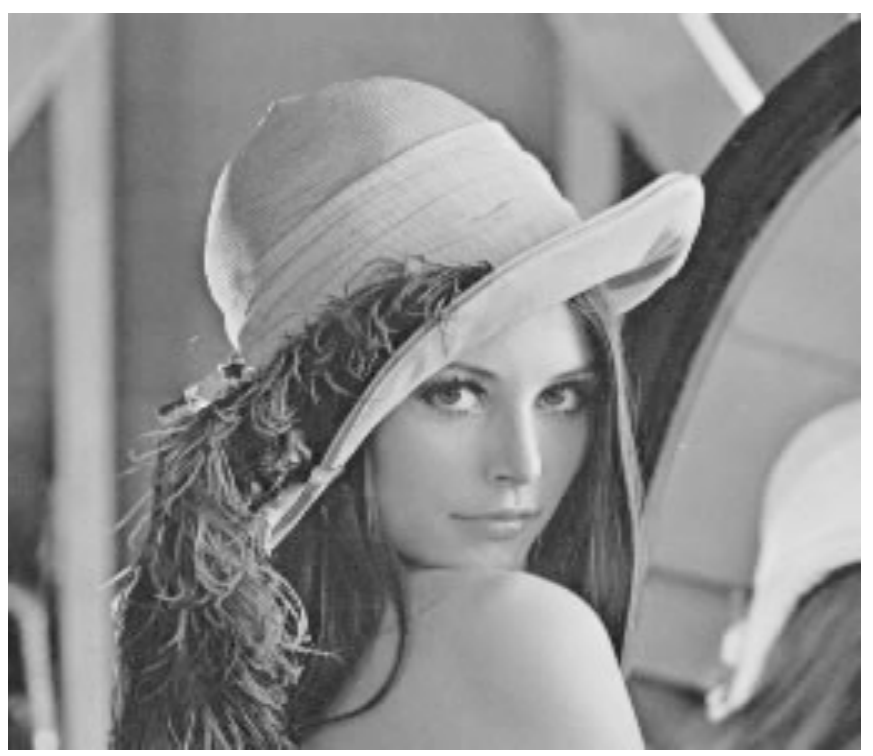

Fig. (7). Original image.

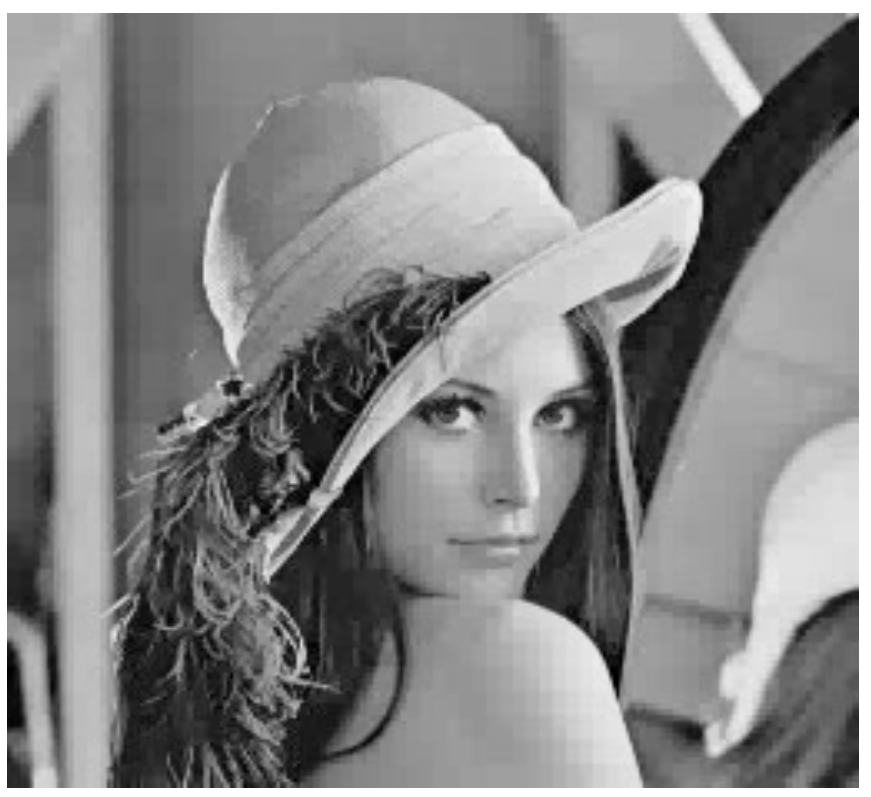

Fig. (8). Resulting image using standard deviation thresholding.

Using higher dimension images, the performance tends to increase and this can be observed in Fig. (12). Compression ratio tends to increase for all the thresholding algorithms when bigger image sizes are used. The PSNR also increases noticeably for the mean and the standard variation thresholds, while for the other four algorithms the PSNR differences are at low levels. Analyzed images are composed by 256 grayscale levels ( 8 bits-per-pixel resolution) so an 


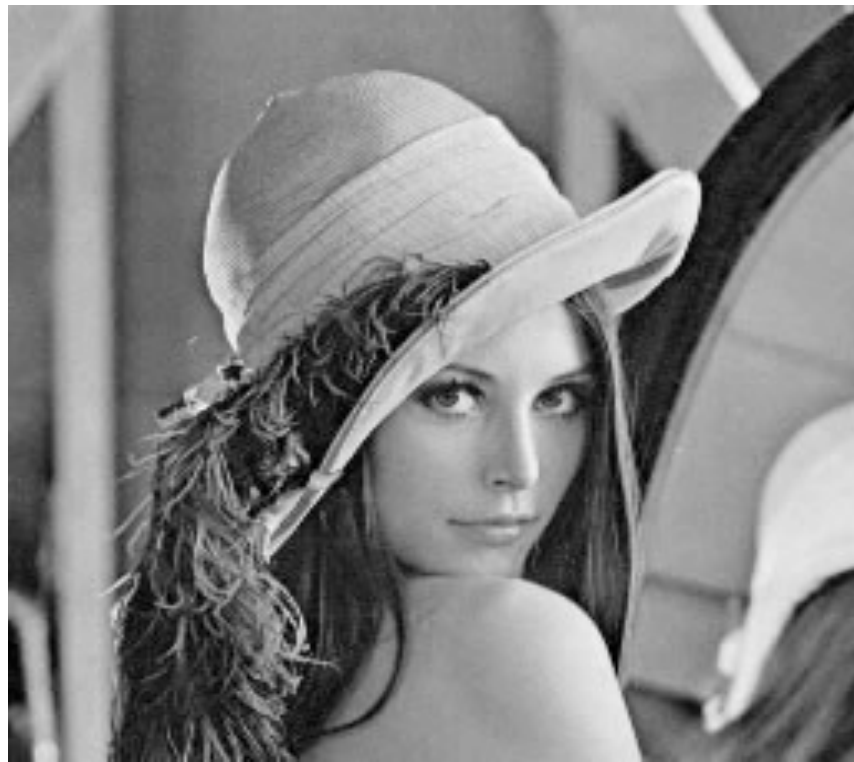

Fig. (9). Resulting image using rigorous sure thresholding.

analysis for color images can be implemented using this method for each of the RGB component matrixes, achieving similar compression ratios and PSNR values. Note that the 64-point vector transform used in this work follows a scan with a Hilbert fractal curve and it therefore allows lower computational time than the conventional $8 \times 8$ sub-images matrix transform. The processing time for the proposed approach is thus in the order of seconds (depending on hardware and image characteristics).

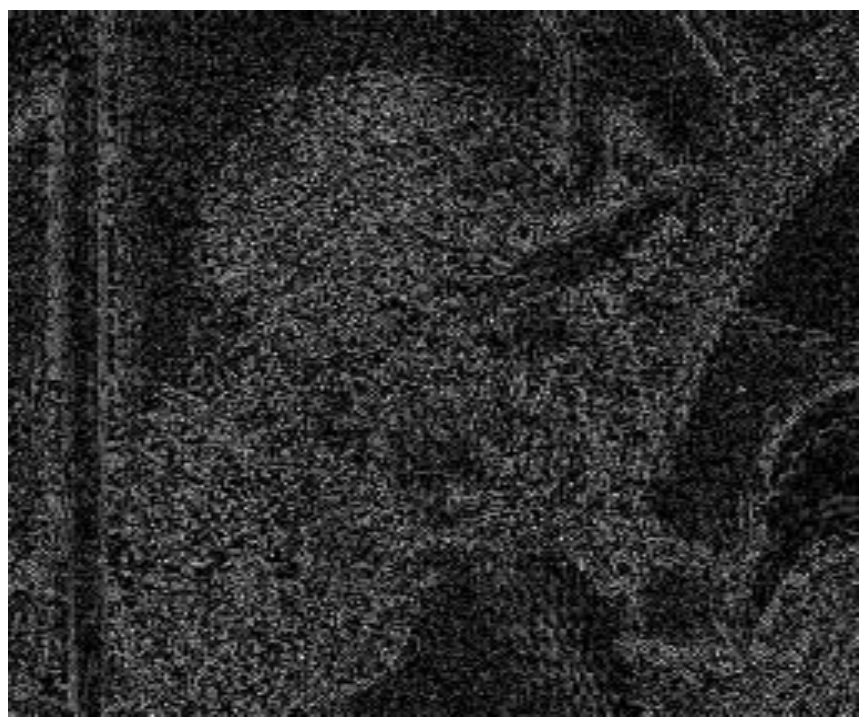

Fig. (10). Error image using standard deviation thresholding.

\section{CONCLUSIONS}

An approach for image compression has been proposed and implemented based on the DWT and several thresholding techniques. Numerical results have been obtained based on high frequency coefficient elimination following different thresholding algorithms. The discrete wavelet transform used achieved good PSNR values. A Hilbert fractal curve is used for sub-image 2-D to 1-D decomposition due to the fact that follows a more distributed

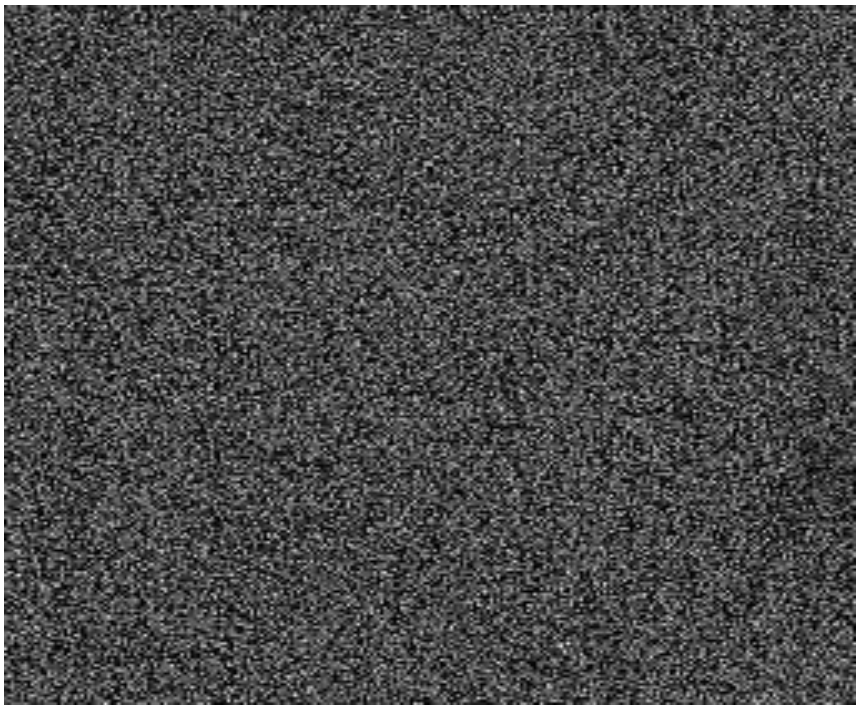

Fig. (11). Error image using rigorous sure algorithm.

a) $R_{C}$

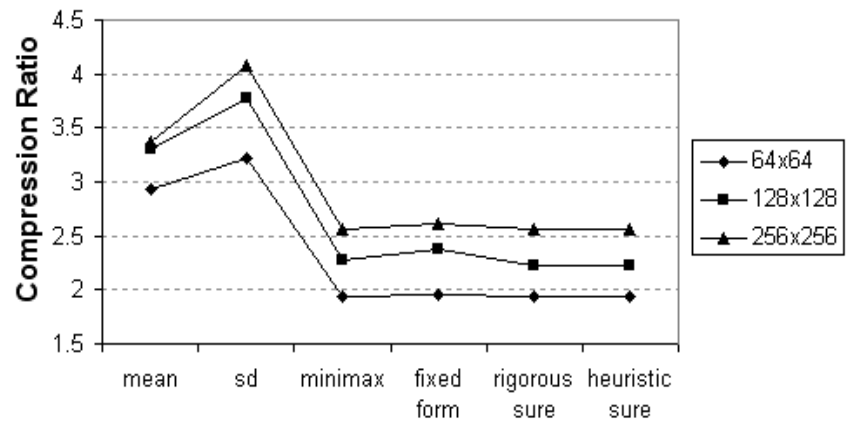

b) $\operatorname{PSNR}(\mathrm{dB})$

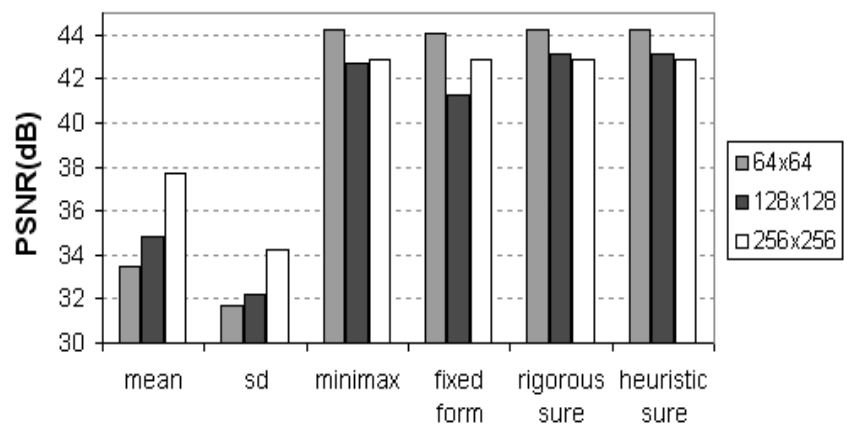

Fig. (12). The values of a) $R_{C}$, and b) PSNR (dB) for different image sizes.

pattern than a linear scan and allows a faster computational performance for those realized by 2-D wavelet transforms. High frequency mean and standard deviation thresholding algorithms generated high compression ratios while the PSNR obtained is low. On the other hand, minimax, fixed form, rigorous sure and heuristic sure algorithms achieved higher PSNR but at lower compression ratios. Performance is better when higher levels of decomposition are used (5level wavelet transforms instead of 3 or 4). Furthermore, a comparison using four different types of mother wavelets has been presented and results have shown that the simpler wavelet is used, better performance was obtained, and this is related to the number of vanishing moments used in wavelet 
functions. The present proposed approach can be used for other purposes, such as image de-noising, where the noise elements are located in high frequency bands or along the entire signal, image border detection and micro-calcifications detection. Wavelet transforms represent an excellent tool in image processing, increasing results in quality and performance.

\section{CONFLICT OF INTEREST}

The authors confirm that this article content has no conflict of interest.

\section{ACKNOWLEGEMENTS}

The authors gratefully acknowledge the financial support from the National Council for Science and Technology of Mexico.

\section{REFERENCES}

J. G. Proakis, Digital Communications, $4^{\text {th }}$ ed. Mc Graw Hill, NY, 2001.

[2] R. C. Gonzalez and R. E. Woods, Digital Image Processing, $2^{\text {nd }}$ ed. Prentice Hall, USA, 2001

[3] K. W. Chen, C. W. Lin, T. H. Chiu, M. Y. Chen, and Y. P. Hung, "Multi-resolution design for large-scale and high-resolution monitoring". IEEE Trans. Multimed., vol. 13, no. 6, 1256-1268, Dec. 2011.

[4] O. Starostenko, V. Alarcon-Aquino, H. Lobato-Morales, and O. Sergiyenko, "Computational Approaches to Support Image-Based
Language Learning Within Mobile Environment”, In: [Special Issue on Mobile and Ubiquitous Technologies for Language Learning], Int. J. Mob. Learn. Org., vol. 4, no. 2, 150-171, Mar. 2010.

[5] G. Yang, N. Zheng, C. Li and S. Guo. "Extensible JPEG2000 image compression systems". In: IEEE International Conference on Industrial Technology, 2005, pp. 1376-1380.

[6] K. H. Talukder and K. Harada. "Development and performance analysis of an adaptive and scalable image compression scheme with wavelets". In: International Conference on Information and Communication Technology, March 2007, pp. 250-253.

[7] J. C. Galan-Hernandez, V. Alarcon-Aquino, O. Starostenko, J. M. Ramirez-Cortes. Foveated ROI compression with hierarchical trees for real-time video transmission. In: Proceedings of the 3rd Mexican Conference on Pattern Recognition, MCPR'11, SpringerVerlag, Berlin, Heidelberg, pp. 240-249, 2011.

[8] V. Alarcon-Aquino and J. A. Barria, "Change detection in time series using the maximal overlap discrete wavelet transform", Latin Am. Appl. Res. An. Int. J., vol. 39, no. 2, 145-152, April 2009.

[9] I. Daubechies, Ten Lectures on Wavelets. New York: SIAM, Philadelphia, 1992.

[10] S. Mallat, A wavelet tour of signal processing. Academic Press, USA, $2^{\text {nd }}$ ed, 1999

[11] S. Sardy, A. Antoniadis, P. Tseng. Automatic smoothing with wavelets for a wide class of distributions. J. Comput. Graph. Stat., vol. 13, no. 2, 399-421, 2004.

[12] J. Qian, Denosing by Wavelet Transform. Rice University, Department of Electrical Engineering, Houston, 2000.

[13] V. Crnojevic, V. Senk and Z. Trpovski, "Lossy lempel-ziv algorithm for image compression", In: 6th International Conference on Telecommunications in Modern Satellite, Cable and Broadcasting Service, TELSIKS, 2003, pp. 522-525.

(C) Alarcon-Aquino et al.; Licensee Bentham Open.

This is an open access article licensed under the terms of the Creative Commons Attribution Non-Commercial License (http://creativecommons.org/licenses/ by-nc/3.0/) which permits unrestricted, non-commercial use, distribution and reproduction in any medium, provided the work is properly cited. 